\title{
APROXIMAÇÕES SOBRE PRODUÇÃO, PRODUTIVIDADE E COLABORAÇÃ̃O CIENTÍFICA ENTRE OS DEPARTAMENTOS DE CIÊNCIA DA INFORMAÇÃO DO ESTADO DE SÃO PAULO
}

\author{
APPROACHES ON PRODUCTION, PRODUCTIVITY AND SCIENTIFIC COLLABORATION \\ AMONG THE SÃO PAULO STATE INFORMATION SCIENCE DEPARTMENTS
}

\author{
Pedro Ivo Silveira Andretta ${ }^{1}$ \\ Eduardo Graziosi Silva ${ }^{2}$ \\ Renan Carvalho Ramos ${ }^{3}$
}

\begin{abstract}
RESUMO
Os indicadores de produção científica são valorizados como forma de mensurar e avaliar parte do desenvolvimento científico de uma área do conhecimento ou região. Esta pesquisa demonstra o perfil de pesquisa de Departamentos de Ciência(s) da Informação das Instituições de Ensino Superior (IES) do Estado de São Paulo (SP), considerando variáveis como produção, produtividade e colaboração científica entre os anos de 2000 a 2009. Conclui-se que a Unesp detém mais colaborações intradepartamental e interinstitucional, além de maior produção; e a USP maior produtividade, apesar de poucas colaborações, sendo constatada ainda a influência dos Programas de Pós-Graduação sobre esses índices.
\end{abstract}

\section{PALAVRAS-CHAVE}

Bibliometria. Ciência da informação. Produtividade e publicações. Colaboração. Métodos, técnicas e ferramentas.

\section{ABSTRACT}

Scientific production indicators are valorized as a way to measure and evaluate part of the scientific development in a given area of expertise. This paper shows the research profile of Information Science Departments from institutions of higher education in São Paulo State, considering variables such as production, productivity and scientific collaboration between the years 2000 and 2009. It has been found that UNESP holds more inter-department and inter-institution collaboration, in addition to greater production; and that USP holds more productivity, in spite of fewer collaborations, being further evidenced the influence cast upon these indexes by Postgraduate programs.

\section{KEYWORDS}

Bibliometrics. Information science. Productivity and publications. Collaboration. Methods, techniques and tools.

\footnotetext{
${ }^{1}$ Mestrando em Linguística - UFSCar Bacharel em Biblioteconomia e Ciência da Informação - UFSCar. E-mail: andretta_pedro@yahoo.com.br.

${ }^{2}$ Graduando em Biblioteconomia e Ciência da Informação - UFSCar. E-mail: eduardograziosi@ig.com.br.

${ }^{3}$ Bacharel em Biblioteconomia e Ciência da Informação pela UFSCar Mestrando do Programa de Pós-Graduação em Ciência, Tecnologia e Sociedade da UFSCar Bibliotecário da UNESP/Rio Claro E-mail: renan@nit.ufscar.br.
} 


\title{
INTRODUÇÃO
}

Cada vez mais se valorizam os indicadores de produção científica como forma de mensurar e avaliar parte do desenvolvimento científico de uma área do conhecimento, de uma dada região ou até mesmo de um país. Além disso, os indicadores podem subsidiar a tomada de decisão no âmbito das políticas científicas e tecnológicas.

\begin{abstract}
Quanto mais ativo e produtivo o ambiente científico, mais freqüentes e rigorosas são as rotinas de avaliação vigentes. Estes processos avaliativos se fundamentam, principalmente, em duas metodologias: a avaliação qualitativa, feita pelos pares, fortemente ancorada na reputação adquirida pelo avaliado; e a que se deriva de critérios quantitativos, baseados em métodos bibliométricos e cientométricos. (VANZ; STUMPF, 2010, p. 67).
\end{abstract}

Os estudos sobre os métodos para a elaboração de indicadores quantitativos da produção e colaboração científica continuam em expansão. No Brasil, essa necessidade de desenvolvimento fez com que o país trabalhasse na elaboração de diferentes tipos de bases de dados que permitissem a elaboração de indicadores, como a biblioteca eletrônica de periódicos $\mathrm{SciELO}^{4}$ e a Plataforma Lattes 5 . (VANZ; STUMPF, 2010).

Estudos quantitativos permitem estabelecer indicadores, que são retratos sempre parciais da realidade. Desse modo, é objetivo desta pesquisa gerar e analisar indicadores de produção, produtividade e colaboração que podem esboçar o atual comportamento de cada Departamento de Ciência(s) da Informação das Instituições de Ensino Superior (IES) do Estado de São Paulo, quando se trata do tema "Pesquisa", ou no seu limite "Publicações", definido por Spinak como "informação em formato permanente para uso comum" (1998, p. 141, tradução do autor), entre os anos de 2000 a 2009. A fim de complementar os indicadores, considerar-se-á dados qualitativos, como as diferenças entre as instituições públicas e privadas, as possibilidades de interação e colaboração, assim como a tradição e cultura de cada instituição.

Para o cumprimento do objetivo desta pesquisa foi adotada como fonte de dados para análise a Plataforma Lattes, já tratada por Lane (2010) como um importante sistema para a

\footnotetext{
${ }^{4}$ SciELO é a sigla utilizada para Scientific Library Online, que é uma biblioteca eletrônica virtual que abrange uma coleção selecionada de revistas acadêmicas brasileiras e da América Latina. (GREENE, 2000). Está disponível em: http://www.scielo.org/php/index.php

${ }^{5}$ O principal componente da Plataforma Lattes é o sistema de Currículo Lattes, um sistema de informação curricular mantido pelo Conselho Nacional de Desenvolvimento Científico e Tecnológico (CNPq). Segundo Alves, Yanasse e Soma (2009) o sistema armazena em torno de 1.140 .000 currículos de pesquisadores e tem elevado potencial para extração da informação. A Plataforma Lattes está disponível em: http://lattes.cnpq.br/
} 
inferência e avaliação do desempenho da ciência, à medida que registra a vida científica de pesquisadores, servindo como um instrumento indispensável à análise de mérito e competência dos projetos apresentados às agências de fomento brasileiras. Ainda sobre a justificativa do emprego da Plataforma Lattes como fonte de dados válida para uso da comunidade científica e para o fazer ciência pode-se mencionar os mais de 40 trabalhos que o utilizaram com diferentes propósitos e métodos nas mais diversos domínios do conhecimento, como se pode ver no SciELO.

No domínio da Ciência da Informação há muitas pesquisas relacionadas a diferentes fins com o uso do currículo Lattes como fonte de dados, dentre essas a de Pinto e González (2010), sobre o comportamento de alguns dos principais autores desta área científica, averiguando onde publicam; Bufrem, Gabriel Junior e Gonçalves (2010) analisaram diacronicamente a questão da colaboração entre pesquisadores vinculados a programas de pós-graduação em Ciência da Informação nos últimos 20 anos; Oliveira (2009) estudou os grupos de pesquisa brasileiros e Noronha et al. (2009) verificaram onde atuam os doutores formados nesta área. Além dessas, destacam-se a dissertação de Marinho (2007) e a tese de Silva (2007) que versaram, respectivamente, sobre a produtividade dos programas de pós-graduação em Ciência da Informação levantando a questão sobre a contabilização de resumos e artigos em anais na pontuação do Qualis nos programas de pós; e os problemas da natureza aberta do currículo Lattes, apontando sugestões para aprimoramento do sistema.

Como visto, este trabalho se diferencia dos demais tanto quanto ao recorte geográfico e temporal, bem como metodológico para analisar a produção, produtividade e colaboração na Ciência da Informação por meio dos currículos Lattes.

\section{METODOLOGIA}

Como mencionado anteriormente, foram muitas as pesquisas realizadas em diferentes domínios da ciência sobre a questão da produção, produtividade e colaboração envolvendo a análise de currículos Lattes. Em comum, essas pesquisas pautaram-se sempre em uma análise manual dos dados, isto é, coleta e análise de dados de currículo a currículo ou, quando muito, um tratamento em um banco de dados construído exclusivamente para trabalhar os dados após a coleta manual. Para a realização desta pesquisa, optou-se por uma inovação tecnólogica e, por conseguinte, metodólogica, considerando ainda seus riscos inerentes. Assim, foram definidas quatro etapas, expostas abaixo: 
1. Mapeamento das IES do Estado de São Paulo que possuem Departamento(s) de Ciência(s) da Informação através do Portal do Ministério da Educação, identificando seus docentes a partir dos sites institucionais;

2. Levantamento dos currículos Lattes dos atuais docentes que lecionam nos Departamentos de Ciência(s) da Informação anteriormente mapeados;

3. Análise dos conjuntos de Currículos Lattes dos docentes por IES, assim como do conjunto de todos do Estado de São Paulo entre os anos de 2000 e 2009, através do software scriptLattes V6.12, considerando as publicações e produção técnica;

4. Tabulação, comparação e descrição dos resultados das análises individuais e geral.

Mena-Chalco e Cesar Junior (2009, p. 32) apontam que o software scriptLattes gera relatórios automaticamente a partir de informações extraídas dos currículos Lattes dos pesquisadores por meio de um sistema de código aberto executado no sistema operacional GNU/Linux. Assim, considerando

[...] um grupo de pesquisadores cadastrados na plataforma Lattes, o scriptLattes baixa os currículos Lattes do site do CNPq, extrai a informação de interesse, elimina a redundância de produções científicas e cria relatórios sobre produção, relatórios de supervisões acadêmicas bem como o grafo de colaboração e o mapa de pesquisa dos membros do grupo. (MENA-CHALCO; CESAR JUNIOR, 2009, p. 32, tradução nossa).

Ressalta-se, ainda, que o scriptLattes é composto de seis módulos ${ }^{6}$ :

1. Seleção de Dados, em que é feito o download dos currículos no formato HTML;

2. Processamento de Dados, que lista as produções bibliográficas, técnicas e artísticas de cada membro do grupo;

3. Tratamento de Redundância, que identifica as informações duplicadas, resultado das colaboração entre pesquisadores;

4. Geração do Grafo de Colaboração, que representa, graficamente, a colaboração entre pesquisadores a partir de suas produções científicas;

5. Geração do Mapa de Pesquisa, que representa a localização geográfica dos membros de um grupo de pesquisa e pode ser criado externamente ao software;

\footnotetext{
${ }^{6}$ No processo de trabalho com os dados, o scriptLattes utiliza os seis módulos de maneira automática, entretanto, isso não garante, segundo os próprios desenvolvedores, que os resultados fornecidos não estejam sujeitos a falhas, que podem ocorrer devido à inconsistências no preenchimento dos currículos.
} 
6. Criação de Relatórios, que gera os relatórios provenientes das produções e supervisões acadêmicas.

\section{RESULTADOS}

Foram mapeadas quatro IES privadas: Centro Universitário Assunção - UNIFAI, Faculdades Integradas Coração de Jesus (FAINC), Pontifícia Universidade Católica de Campinas (PUC-Campinas), Faculdades Integradas Teresa D`Ávila (FATEA), das quais somente a última possuía no site institucional os nomes dos docentes; e três públicas, Universidade Federal de São Carlos (UFSCar), Universidade Estadual Paulista Júlio de Mesquita Filho (Unesp), Universidade de São Paulo (USP) campus de São Paulo e campus de Ribeirão Preto, sendo que os docentes do curso de Ciências da Informação e Documentação da USP do campus de Ribeirão Preto estavam inseridos no Departamento de Física e Matemática e, por isso, não foram considerados neste estudo.

Desse modo, foram considerados para o quadro de análise os currículos dos professores apresentados nos sites institucionais em agosto de 2010. Sobre os dados analisados, ressalva-se a não observação da data de inserção do docente no departamento, o histórico de desligamentos ou, ainda, as ocasionais desatualizações dos currículos ${ }^{7}$ analisados.

Após a identificação dos docentes e seus respectivos currículos Lattes, processaram-se cinco análises, via scriptLattes, envolvendo, a princípio separadamente, os docentes da FATEA, UFSCar, USP e Unesp e depois outra com todos, com vistas à obtenção da análise do Estado de São Paulo $^{8}$. A listagem dos currículos envolvidos nesse estudo pode ser vista no Apêndice 1 - Lista dos Currículos Analisados.

As informações fornecidas pelo scriptLattes foram sistematizadas em planilhas com o propósito de organizar os dados relativos à produção acadêmica e obter índices de produtividade e participação percentual, obtendo os seguintes quadros com suas respectivas análises.

QUADRO 1

Publicações por docentes Departamentos de CI no Estado de São Paulo (2000-2009)

$$
\text { QUANTIDADES DE PUBLICAÇÕES (2000 - 2009) }
$$

\begin{tabular}{|l|l|l|l|l|}
\hline FATEA & UFSCar & Unesp & USP & $\begin{array}{l}\text { ESTADO } \\
\text { SP }\end{array}$ \\
\hline
\end{tabular}

\footnotetext{
${ }^{7}$ O CNPq realiza a cada dois anos censos através dos currículos Lattes e solicita aos portadores de currículo nesse sistema sua constante atualização, inclusive para a concessão de financiamento para pesquisas.

${ }^{8}$ Os relatórios gerados por meio do SciptLattes estão disponíveis em:

<http://www.4shared.com/file/MeZV8K3c/DCIs.html >
} 


\begin{tabular}{|l|l|l|l|l|l|}
\hline Artigos completos publicados em periódicos & 39 & 105 & 157 & 140 & 432 \\
\hline Livros publicados/organizados ou edições & 1 & 24 & 27 & 73 & 124 \\
\hline $\begin{array}{l}\text { Capítulos de livros publicados } \\
\text { Textos em jornais de notícias/revistas publicados em anais de }\end{array}$ & 18 & 77 & 161 & 118 & 364 \\
\hline $\begin{array}{l}\text { Trabalhos completos anais de } \\
\text { congressos }\end{array}$ & 124 & 215 & 314 & 230 & 772 \\
\hline $\begin{array}{l}\text { Resumos expandidos publicados em } \\
\text { congressos }\end{array}$ & 71 & 62 & 10 & 143 \\
\hline $\begin{array}{l}\text { Resumos publicados em anais de congressos } \\
\text { Apresentações de trabalho }\end{array}$ & 21 & 70 & 267 & 32 & 387 \\
\hline $\begin{array}{l}\text { Demais tipos de produção bibliográfica } \\
\text { Total de publicações }\end{array}$ & 12 & 33 & 434 & 275 & 841 \\
\hline
\end{tabular}

FONTE - Própria.

Como se nota, a FATEA tem a menor produção de publicação, equiparando-se a apenas à UFSCar em quantidade de textos publicados em jornais de revistas e notícias. Tal fato deve-se, também, a sua natureza de instituição privada, visto que seu foco recai sobre a formação de profissionais para o mercado de trabalho, isto é, está mais voltada para atividades de ensino do que atividades relacionadas à pesquisa e à extensão. Porém, ressalta-se que a FATEA possui um Núcleo de Extensão Universitária, que desde 2005 " [...] tem trabalhado na disseminação do conceito de extensão universitária para os coordenadores dos cursos academicos (sic) e discentes e na solicitação de suas participações nos projetos sociais.” (APRESENTAÇÃO, 2011). Futuramente, portanto, a participação de docentes e discentes no Núcleo poderá alterar a quantidade de publicações atuais, decorrente da divulgação dos resultados alcançados nas atividades extensionistas.

A UFSCar apresenta vantagem superior sobre as demais instituições em resumos expandidos publicados em anais de congresso. Sobre isso, pode-se supor que a preferência dos pesquisadores dessa instituição pelos resumos expandidos decorre da agilidade de divulgação dos resultados tanto de pesquisas em andamento como de pesquisas concluídas.

A Unesp, por sua vez, lidera na produção de artigos completos publicados em periódicos, capítulos de livros, textos completos e resumos publicados em anais de congresso e na quantidade total de publicações. Essa situação deve-se não só a realização contínua de pesquisas, impulsionadas pela realização de atividades de pesquisa e extensão nos cursos de Bacharelado em Biblioteconomia, Bacharelado em Arquivologia e Programa de Pós-Graduação em Ciência da Informação nos níveis de Mestrado e Doutorado, mas também pelo fato de ser, dentre as 
instituições analisadas, aquela que conta com o maior número de docentes, conforme apresentado no Apêndice 1.

A USP tem maior produção vinculada à organização e edição de livros, textos em jornais de notícias além de outros tipos de produção bibliográfica. Deste modo, percebe-se a preferência dessa instituição por uma forma de comunicação científica típica das Ciências Sociais Aplicadas, especificamente das Ciências Humanas: a publicação de livros. Ainda em relação às demais instituições, a USP lidera a produção de textos em jornais de notícias, contribuindo para a divulgação da Ciência da Informação na sociedade.

A participação percentual da produção científica dos Departamentos de Ciência(s) da Informação pode ser vista no quadro a seguir.

\section{TABELA 2}

Participação percentual de publicações nos Dept. de CI do Estado de São Paulo

\begin{tabular}{|l|l|l|l|l|}
\hline $\begin{array}{l}\text { Participação em \% de publicações nos } \\
\text { Dept. de CI no Estado de São Paulo (2000-2009) }\end{array}$ & FATEA & UFSCar & Unesp & USP \\
\hline Artigos completos publicados em periódicos & 9,03 & 24,31 & 36,34 & 32,41 \\
\hline Livros publicados/organizados ou edições & 0,81 & 19,35 & 21,77 & 58,87 \\
\hline Capítulos de livros publicados & 4,95 & 21,15 & 44,23 & 32,42 \\
\hline Textos em jornais de notícias/revistas & 4,78 & 4,78 & 16,73 & 73,71 \\
\hline Trabalhos completos publicados em anais de congressos & 3,11 & 27,85 & 40,67 & 29,79 \\
\hline Resumos expandidos publicados em anais de congressos & 0,70 & 49,65 & 43,36 & 6,99 \\
\hline Resumos publicados em anais de congressos & 5,43 & 18,09 & 68,99 & 8,27 \\
\hline Apresentações de trabalho & 2,02 & 14,27 & 51,61 & 32,70 \\
\hline Demais tipos de produção bibliográfica & 2,82 & 7,75 & 30,75 & 59,62 \\
\hline Total de publicações & 3,87 & 19,43 & 42,65 & 35,22 \\
\hline
\end{tabular}

FONTE - Própria.

Os valores acima estão relacionados à produção, isto é, em valores brutos. Isso posto, pondera-se que a existência ou não de Programas de Pós-Graduação Acadêmicos, mantidos pelos departamentos nas instituições apresentadas, influenciam de maneira bastante significativa nos dados relacionados à produção científica. Nesse contexto, é válido ressaltar que a FATEA não possui nenhum Programa de Pós-GraduaçãoAcadêmico, enquanto que na UFSCar, o Departamento de Ciência da Informação mantêm o recente Programa de Pós-Graduação em Ciência, Tecnologia e Sociedade, de caráter multidisciplinar, oferecido no nível Mestrado desde 2007. A Unesp e a USP, mantém por meio de seus Departamentos de Ciência da Informação, os Programas de Pós- 


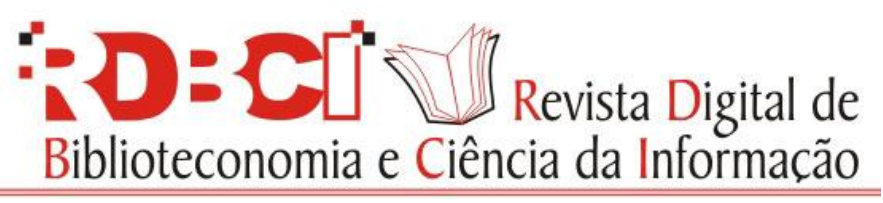

ARTIGO

Graduação em Ciência da Informação desde 2001 e 2006, respectivamente, ambos nos níveis Mestrado e Doutorado. No caso da USP, ressalta-se que essa instituição detinha, até a década de 1990, o Programa de Pós-Graduação em Ciências da Comunicação com uma linha de concentração intitulada primeiramente de "Biblioteconomia", depois de 1997 "Ciência da Informação e Documentação" e, em 2006 esta ganha autonomia, tornando-se o Programa de Pós-Graduação em Ciência da Informação". Assim, a tradição dos Programas de Pós-Graduação Acadêmica em Ciência da Informação na Unesp e na USP contribuíram para seus elevados índices de participação em porcentagem de publicações, enquanto que a FATEA e a UFSCar, por não contarem com a existência de um Programa de Pós-Graduação de ambito acadêmico, no primeiro caso, e pelo fato de ter sido criado recentemente como no segundo caso, não apresentam índices elevados como das demais instituições. Outra forma de ver a performance de uma instituição é por meio da produtividade (entendida aqui como o quociente entre a quantidade de publicações e a quantidade de docentes que atuam nos departamentos análisados e listados no Apêndice 1), cujos indicadores são apresentados no quadro a seguir. Observa-se que as células em vermelho indicam os valores menores que a média estadual, e as em azul, maiores que a média.

TABELA 3

Produtividade nas Publicações por docentes em Dept. de CI no Estado de São Paulo (2000-2009)

\begin{tabular}{|c|c|c|c|c|c|}
\hline \multicolumn{6}{|l|}{$\begin{array}{l}\text { Produtividade nas Publicações por docentes em } \\
\text { Depts. de CI no Estado de São Paulo (2000-2009) }\end{array}$} \\
\hline & FATEA & UFSCar & Unesp & USP & \begin{tabular}{|l} 
ESTADO \\
SP \\
\end{tabular} \\
\hline Artigos completos publicados em periódicos & 3,25 & 6,56 & 7,48 & 8,24 & 6,55 \\
\hline Livros publicados/organizados ou edições & $\mathbf{0 , 0 8}$ & 1,5 & 1,29 & 4,29 & 1,88 \\
\hline Capítulos de livros publicados & 1,5 & 4,81 & 7,67 & 6,94 & 5,52 \\
\hline Textos em jornais de notícias/revistas & 1 & 0,75 & 2 & 10,88 & 3,8 \\
\hline $\begin{array}{l}\text { Trabalhos completos publicados em anais de } \\
\text { congressos }\end{array}$ & 2 & 13,44 & 14,95 & 13,53 & 11,7 \\
\hline $\begin{array}{l}\text { Resumos expandidos publicados em anais de } \\
\text { congressos }\end{array}$ & 0,08 & 4,44 & 2,95 & 0,59 & 2,17 \\
\hline Resumos publicados em anais de congressos & 1,75 & 4,38 & 12,71 & 1,88 & 5,86 \\
\hline Apresentações de trabalho & 1,42 & 7,5 & 20,67 & 16,18 & 12,74 \\
\hline Demais tipos de produção bibliográfica & 1 & 2,06 & 6,24 & 14,94 & 6,45 \\
\hline Total de publicações & 12,08 & 45,44 & 76 & 77,53 & 56,7 \\
\hline
\end{tabular}

FONTE - Própria. 
Como se nota, os maiores índices no Estado de São Paulo são, respectivamente: apresentação de trabalhos, trabalhos completos publicados em anais de congresso, artigos completos publicados em periódicos e demais tipos de produção bibliográfica. A FATEA apresenta indicadores críticos de produtividade, assim como de produção; isso se deve, como apontado anteriormente, pelo fato dessa instituição ser de natureza privada, tendo como foco o ensino, embora conte com atividades de pesquisa e de extensão apoiadas por seu Núcleo de Extensão.

Considerando as instituições de ensino públicas, pode-se dizer que a UFSCar possui os índices regulares de artigos completos publicados em periódicos, trabalhos completos publicados em anais de congressos e resumos expandidos publicados em anais de congressos, sendo líder apenas da produção desse último. A Unesp, por sua vez, possui bons índices de produtividade, liderando a produção de capítulos de livros, trabalhos completos e resumos publicados em anais de congressos e por conseguência apresentação de trabalhos, produzindo menos organizações de livros, textos em jornais de notícias/revistas e demais tipos de produção bibliográfica. A USP, apesar de não possuir a maior produção de artigos de periódicos, tem nessa modalidade a maior produtividade; sendo a instituição que menos tem indicadores críticos em relação a média de produtividade do estado de São Paulo, sendo raro a produção de resumos e resumos expandidos publicados em anais de congressos.

A colaboração científica entre os departamentos de Ciência da Informação do estado de São Paulo é apresentada no grafo a seguir. Vale ressaltar que o grafo é gerado automaticamente pelo ScriptLattes em grande dimensão, disso a dificuldade em vê-lo em pequena escala, e a aproximação entre os atores, ou nós, não é bem explicada pelos desenvolvedores do software: 


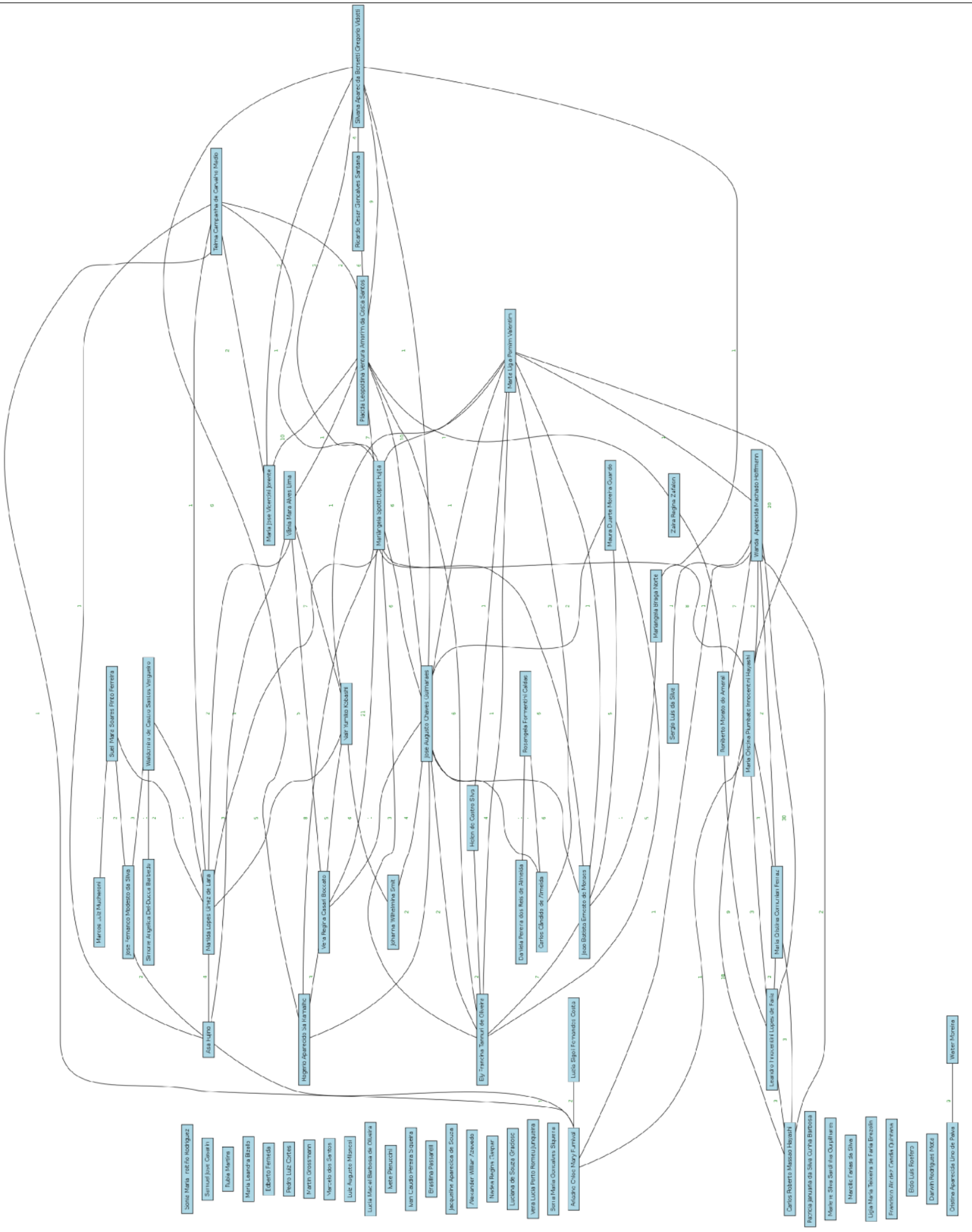

FIGURA 1 - Grafo de colaboração

FONTE - ScriptLattes V6.12 
Considerando a dificuldade em perceber as colaborações intradepartamentais e interinstitucionais, foram contabilizados manualmente os relacionamentos do grafo de colaboração gerado automaticamente pelo ScriptLattes, isto é, sem qualquer ambição de uma análise de redes sociais com seus cálculos de densidade, centralidade, e intermediação entre os atores da rede; mas cujo objetivo é apenas a melhor observação dos dados da Figura 1.:

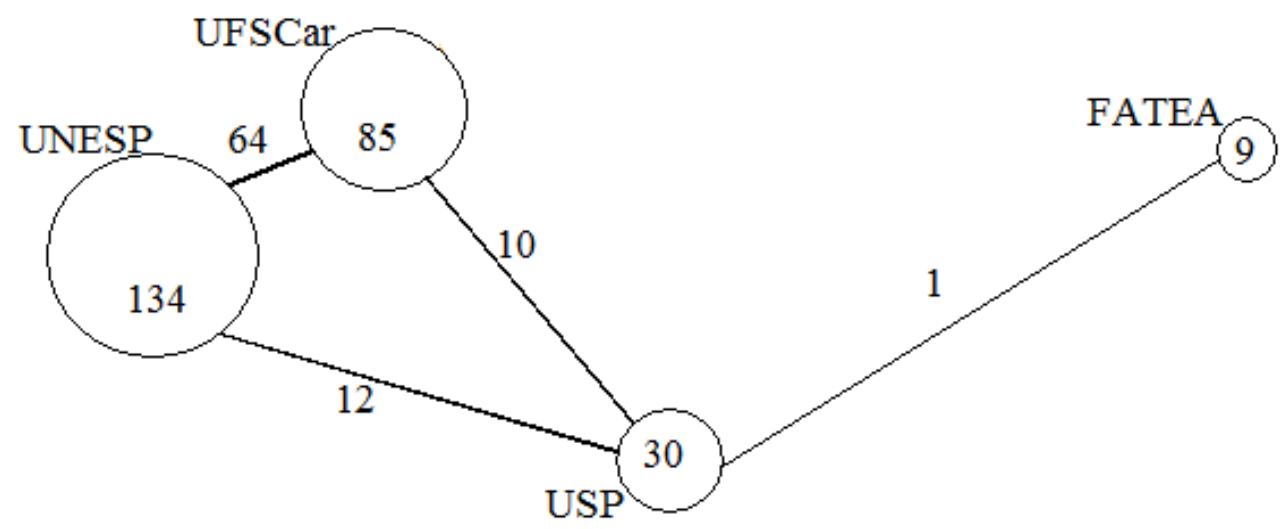

FIGURA 2 - Grafo de colaboração simplificado FONTE - Própria.

Percebe-se que há uma forte rede de colaboração entre os docentes da Unesp tanto interna (intradepartamental) como externa (interinstitucional), seguida da UFSCar. Isso se deve, dentre outros fatores, pelo fato de alguns docentes da UFSCar participarem do Programa de PósGraduação em Ciência da Informação, nos níveis Mestrado e Doutorado, na Unesp, que por meio da criação de parcerias, desenvolvimento conjunto de pesquisas, realização de eventos, dentre outras atividades acadêmicas, contribui para o aumento do índice de colaboração entre os pesquisadores dessas instituições. A USP, por sua vez, mantém poucas colaborações internas e externas, sendo a única com um trabalho em comum com a FATEA e com um índice de colaboração semelhante tanto com a Unesp quanto com a UFSCar. Ressalta-se que na Figura 2, a posição central da USP justifica-se pelo fato de ter tido contato com todas as instituições analisadas, embora apresente um número menor de colaborações. A FATEA, por sua vez, possui os menores índices de colaboração interna e externa.

Se, por um lado, a Unesp tem mais trabalhos produzidos, sua posição de destaque devese, mas não exclusivamente, às colaborações internas e externas, que podem possibilitar a elaboração de um número maior de trabalhos; por outro lado, a USP tem pouca colaboração interna 
e externa, mas ainda assim guarda para si a posição de instituição mais produtiva, o que não significa, pelo que foi apresentado, que não possua trabalhos em colaboração com outros departamentos paulistas ou instituições fora do Estado de São Paulo.

\section{CONSIDERAÇÕES FINAIS}

Essa pesquisa realizou, por meio de uma abordagem discreta de cunho quantitativo, a análise de indicadores de produção, produtividade e a colaboração entre as Instituições de Ensino Superior que comportam Departamentos de Ciência(s) da Informação no Estado de São Paulo, por intermédio da ferramenta ScriptLattes, com o intuito de poder colaborar com o avanço científico e pedagógico nos cursos de graduação e Pós-Graduação em Ciência(s) da Informação na região. Procurou-se, ainda, enfocar aspectos qualitativos, como a existência de Programas de PósGraduação, a parceria para a realização de eventos acadêmicos, desenvolvimento conjunto de pesquisas, dentre outros.

O ScriptLattes possibilitou estabelecer, exclusivamente, os indicadores de produção dos departamentos (Tabela 2) e de colaboração entre docentes (Figura 1), subsidiando a elaboração de indicadores de produtividade (Tabela 3) e a rede de colaboração entre os departamentos (Figura 2), que foi contada manualmente. Portanto, essa ferramenta revelou-se adequada para o objetivo da pesquisa, possibilitando a obtenção dos indicadores supracitados, que, em conjunto com dados qualitativos, permitiu uma análise panorâmica da produção, produtividade e colaboração dos Departamentos de Ciência da Informação no Estado de São Paulo.

Por meio dos dados coletados e sua respectiva análise, percebeu-se que as instituições públicas lideram os indicadores de produção e de produtividade, interagindo com os relacionamentos de colaboração mais fortes entre si. Já a instituição particular analisada não possui nenhum Programa de Pós-Graduação Acadêmico, o que justifica os indicadores obtidos e comprova de certo modo, que a FATEA não foca tanto seus esforços em pesquisa, mas sim no ensino de graduação. Ressalta-se que a diferença na produção científica entre os departamentos das instituições analisadas, é devida, dentre outros fatores, à existência ou não de Programas de PósGraduação Acadêmicos, e também a tradição desses. 


\section{REFERÊNCIAS}

APRESENTAÇÃO - Curso de Biblioteconomia. Disponível em:

<http://fatea.br/fatea/biblioteconomia/apresentacao/>. Acesso em: 30 set. 2011.

BUFREM, L. S.; GABRIEL JUNIOR, R. F.; GONÇALVES, V. Práticas de co-autoria no processo de comunicação científica na pós-graduação em ciência da informação no Brasil. Informação \& Informação, Londrina, v. 15, n. esp., p. 110-129, 2010. Disponível em: <

http://www.uel.br/revistas/uel/index.php/informacao/article/viewArticle/5506 >. Acesso em: 7 mar. 2010.

GREENE, L. J. Mais visibilidade para a ciência brasileira. Pesquisa FAPESP, São Paulo, n. 57, 2000. Disponível em: <

http://www.revistapesquisa.fapesp.br/index.php?art=1082\&bd=1\&pg=1\&lg >. Acesso em 7 mar. 2011.

LANE, J. Let's make science metrics more scientific. Nature, v. 464, 25 March 2010. p. 488-489. Disponível em: < http://www.nature.com/nature/journal/v464/n7288/full/464488a.html >. Acesso em 05 fev. 2011

MARINHO, I. A comunicação científica e o modelo de comunicação organizacional: análise quantitativa de produtividade dos programas de pós-graduação em Ciência da Informação por meio do Currículo Lattes. 2007. 107 f. Dissertação (Mestrado em Ciência da Informação)-Universidade de Brasília, Brasília, 2007. Disponível em:

< http://repositorio.bce.unb.br/bitstream/10482/3443/1/2007_IracemaMarinho.pdf >. Acesso em: 7 mar. 2011.

NORONHA, D. et al. Egressos dos Programas de Pós-Graduação em Ciência da Informação: por onde andam os doutores?. Perspectivas em Ciência da Informação, Belo Horizonte, v. 14, n. 2, p. 94-107, maio/ago. 2009. Disponível em:

< http://portaldeperiodicos.eci.ufmg.br/index.php/pci/article/view/837/581 >. Acesso em: 7 mar. 2011.

MENA-CHALCO, J. P.; CESAR JUNIOR, R. M. S. ScriptLattes: an open-source knowledge extraction system from the Lattes platform. Journal of the Brazilian Computer.

Society, Campinas, v. 15, n. 4, p. 31-39, 2009. Disponível em:

< www.scielo.br/pdf/jbcos/v15n4/04.pdf >. Acesso em: 7 mar. 2011.

OLIVEIRA, Marlene de. Grupos de Pesquisa em Ciência da Informação no Brasil. Tendências da

Pesquisa Brasileira em Ciência da Informação, João Pessoa, v. 2, n. 1, p. 38-59, 2009.

Disponível em: < http://inseer.ibict.br/ancib/index.php/tpbci/article/view/15/37 >. Acesso em 13 dez. 2010 
PINTO, A. L.; MOREIRO GONZÁLEZ, J. A. Representación productiva de los programas de postgrado en Biblioteconomía y Ciencia de la Información en Brasil: visibilidad en las bases de datos y aspectos del Currículo Lattes de los investigadores. Em Questão, Porto Alegre, v. 16, n. 2 , p. 15-32, jul./dez. 2010. Disponível em: < http://seer.ufrgs.br/EmQuestao/article/view/16073/10448 >. Acesso em: 7 mar. 2011.

SILVA, F. M.; Organização da informação em sistemas eletrônicos abertos de Informação Científica \& Tecnológica: Análise da Plataforma Lattes. 2007. 163 f. Tese (Doutorado em Ciência da Informação) - Departamento de Biblioteconomia e Documentação, Universidade de São Paulo, São Paulo, 2007. Disponível em: < http://www.teses.usp.br/teses/disponiveis/27/27151/tde17032008-095556/publico/lattes.pdf >. Acesso em 13 dez. 2010

SPINAK, E. Indicadores cienciometricos. Ciência da Informação, Brasília, v. 27, n. 2, p. 141148, 1998.

VANZ, S. A. de S.; STUMPF, I. R. C. Procedimentos e ferramentas aplicados aos estudos bibliométricos. Informação \& Sociedade: Estudos, v. 20, n. 2, p. 67-75. Disponível em: < http://www.ies.ufpb.br/ojs2/index.php/ies/article/view/4817 >. Acesso em: 7 fev. 2011. 


\section{APENDICE 1 - LISTA DOS CURRÍCULOS ANALISADOS:}

\section{FATEA}

1.Cristina Aparecida Lino de Paiva

2.Darwin Rodrigues Mota

3.Elcio Luis Roefero

4.Francisco Alcidez Candia Quintana

5.Ligia Maria Teixeira de Faria Brezolin

6.Marcílio Farias da Silva

7.Marlene Silva Sardinha Gurpilhares

8.Patricia Januaria da Silva Cunha Barbosa

9.Simone Angélica Del-Ducca Barbedo

10.Sonia Maria Gonçalves Siqueira

11.Vera Lucia Porto Romeu Junqueira

12.Walter Moreira

\section{UFSCar}

1.Ariadne Chloe Mary Furnival

2.Carlos Roberto Massao Hayashi

3.Leandro Innocentini Lopes de Faria

4.Luciana de Souza Gracioso

5.Luzia Sigoli Fernandes Costa

6.Maria Cristina Comunian Ferraz

7.Maria Cristina Piumbato Innocentini

Hayashi

8.Nádea Regina Gaspar

9.Rogério Aparecido Sá Ramalho

10.Roniberto Morato do Amaral

11.Sergio Luis da Silva

12.Vera Regina Casari Boccato

13.Wanda Aparecida Machado Hoffmann

14.Zaira Regina Zafalon

15.Alexander Willian Azevedo

16.Jacqueline Aparecida de Souza

\section{UNESP}

1.Carlos Cândido de Almeida

2.Daniela Pereira dos Reis de Almeida

\section{Edberto Ferneda}

4.Ely Francina Tannuri de Oliveira

5.Helen de Castro Silva

6.João Batista Ernesto de Moraes

7.José Augusto Chaves Guimaraes

8.Maria José Vicentini Jorente

9.Maria Leandra Bizello

10.Mariangela Braga Norte

11.Mariângela Spotti Lopes Fujita

12.Marta Lígia Pomim Valentim

13.Maura Duarte Moreira Guarido

14.Plácida Leopoldina Ventura Amorim da

Costa Santos

15.Ricardo Cesar Gonçalves Santana

16.Rosangela Formentini Caldas

17.Rúbia Martins

18.Silvana Aparecida Borsetti Gregorio

Vidotti

19.Telma Campanha de Carvalho Madio

20.Samuel José Casarin

21.Sonia Maria Troitiño Rodriguez

USP

1.Asa Fujino

2.Brasilina Passarelli

3.Ivan Claudio Pereira Siqueira

4.Ivete Pieruccini

5.Johanna Wilhelmina Smit

6.Jose Fernando Modesto da Silva

7.Lúcia Maciel Barbosa de Oliveira

8.Luiz Augusto Milanesi

9.Marcelo dos Santos

10.Marcos Luiz Mucheroni

11. Marilda Lopes Ginez de Lara

12.Martin Grossmann

13. Nair Yumiko Kobashi

14.Pedro Luiz Côrtes

15.Sueli Mara Soares Pinto Ferreira

16. Vânia Mara Alves Lima

17. Waldomiro de Castro Santos Vergueiro 